\title{
The development of pandanus tool manufacture in wild New Caledonian crows
}

\author{
Jennifer C. Holzhaider ${ }^{1)}$, Gavin R. Hunt \& Russell D. Gray \\ (Department of Psychology, University of Auckland, Private Bag 92019, Auckland 1142, \\ New Zealand)
}

(Accepted: 17 December 2009)

\begin{abstract}
Summary
New Caledonian crows have remarkably complex tool manufacturing abilities. Here we document the ontogeny of pandanus tool manufacture in wild NC crows. Our results show that the development of wide pandanus tool manufacture is a lengthy process comparable to the development of tool use in primates. Juveniles pass through four main stages of tool manufacture before they acquire adult-like proficiency. By 10 to 12 months of age most juveniles can manufacture tools with adult-like competency, but adult-like speed in manufacture and tool use is only reached in their second year. Whilst individual trial and error learning appears to play a major role in juveniles' development of pandanus tool skills, this development takes place in an environment scaffolded by parental birds. Juveniles stay close to their parents for their first year and have ample opportunity to observe parental tool manufacture and use. Parents influence the juveniles' early learning by leading them to Pandanus sp. trees where they provide discarded tools for early tool use. Exposure to parental tools might help juveniles form a mental template of functional tool design and, thus, facilitate the faithful transmission of local design traditions.
\end{abstract}

Keywords: New Caledonian crows, tool manufacture, social learning, template matching, niche construction, pandanus tools, trial and error.

\section{Introduction}

Basic tool use occurs in many animals from insects to elephants, but complex tool skills are rare (Beck, 1980). One famous example of complex tool skills is the manufacture of stick tools by wild chimpanzees (Pan troglodytes) to

\footnotetext{
1) Corresponding author's e-mail address: jenny.holzhaider@gmail.com 
dip for ants and termites (Goodall, 1968; McGrew, 1974). Tool manufacture by chimpanzees in the Taï Forest usually involves detaching a stick from a tree, modifying its length, and then removing the bark and leaves (Boesch \& Boesch, 1990). Another example is nut cracking, which is considered to be one of the most complex tool behaviours in nonhuman primates because it involves the coordination of three objects (a nut, a hammer and an anvil) (Inoue-Nakamura \& Matsuzawa, 1997). Nut cracking has been observed in West African chimpanzee communities (Whiten et al., 1999, 2001), in tufted capuchin monkeys (Cebus apella) in Brazil (Fragaszy et al., 2004; Ottoni \& Izar, 2008) and in long-tailed macaques (Macaca fascicularis aurea) in South East Asia (Gumert et al., 2009). The variation in behavioural repertoires between populations of both chimpanzees and orangutans has been suggested to reflect different cultures maintained by social learning (e.g., Whiten et al., 1999, 2001; van Schaik et al., 2003).

The New Caledonian crow (Corvus moneduloides, NC crows hereafter) is the only bird species with complex tool skills (Hunt, 1996, 2000a,b; Hunt \& Gray, 2002, 2007). Besides crafting crochet-like hooked tools out of twigs (Hunt \& Gray, 2004a), NC crows manufacture three distinct tool designs from the barbed edges of Pandanus species leaves (Hunt \& Gray, 2003). These three designs vary in complexity from the simple uniformly wide and narrow designs to the more complex stepped, or tapered, design. The three designs have a continuous and overlapping geographical distribution on mainland Grand Terre, with no obvious ecological correlates. Each design is characterized by a high degree of local standardisation. The specific design made at a site can remain the same for decades, suggesting high fidelity transmission (Hunt \& Gray 2003; G.R.H., unpublished data). Hunt and Gray (2003) proposed that the diversification of pandanus tools arose through a process of cumulative technological evolution. However, little is known about the ontogeny of NC crows' pandanus tool skills in the wild, or the extent to which various social learning mechanisms might be involved.

Social learning mechanisms can range in cognitive sophistication from low-level local and stimulus enhancement to high-level imitation. Laboratory experiments have shown that imitation is important in how human children learn tool skills (Want \& Harris, 2001, 2002; Call et al., 2005; Horner $\&$ Whiten, 2005), while nonhuman apes appear more likely to emulate. 
That is, they copy the outcome of an action rather than its exact motor pattern (Tomasello et al., 1987; Nagell et al., 1993; Call \& Tomasello, 1994; Call et al., 2005; Horner \& Whiten, 2005; but see Whiten et al., 2009). It has been claimed that imitation is crucial for the faithful transmission of learnt behaviour and, thus, for the possibility of cumulative cultural evolution (Boyd \& Richerson, 1996; Tomasello, 1999). Others challenge this view (Heyes, 1993; Laland \& Hoppitt, 2003). For example, Heyes (1993) argues that the fidelity of transmission relies on insulating socially transmitted information from individual modification, rather than a particular learning process.

There is little evidence for true imitation in animals in the wild (Laland $\&$ Hoppitt, 2003). Matsuzawa et al. (2001) propose a mechanism of 'education by master-apprenticeship' to describe the social learning process in wild chimpanzee communities. They suggest that in the first 4-5 years of a juvenile's life adults (especially the mother) are highly tolerant and provide young chimpanzees with many opportunities to observe tool use, while the actual motor patterns are learned individually. While not actively teaching, adults allow juveniles to watch actions from close proximity and to scrounge and interact with objects used in the process, thus facilitating the development of tool use (Matsuzawa et al., 2001; Biro et al., 2006). Laboratory experiments confirm the importance of 'leftover' tools in the acquisition of tool using skills by naïve individuals (Hirata \& Morimura, 2000; Hirata \& Celli, 2003). This process can be interpreted as a case of epistemic niche construction, whereby organisms modify the learning environment of their offspring (Laland et al., 2000; Odling-Smee et al., 2003; Sterelny, 2006). This niche construction might lead to the faithful transmission of behaviours in the absence of high-level learning mechanisms such as imitation (Reisman, 2007).

Initial tool use in both humans and nonhuman primates is preceded by a long period of object exploration and learning. Infants in many primate species have a predisposition for certain manipulatory action patterns, such as banging a surface with a held object, that precede using objects as tools (human infants: Thelen \& Smith, 1994; Rochat, 2001; capuchin monkeys: Fragaszy \& Adams-Curtis, 1997; chimpanzees: Takeshita et al., 2005). Infants show all the elements of the behaviour in rudimentary form before assembling them correctly and in the right order to carry out proficient tool use (McGrew et al., 1979; Nishida \& Hiraiwa, 1982; Connolly \& Dalgleish, 1989; de Resende et al., 2008). The development of proficient tool use is also 
an extended process. For example, children require many months to successfully use a spoon to eat (Connolly \& Dalgleish, 1989), and even longer to accomplish more sophisticated tasks. Young chimpanzees at Bossou, as well as tufted capuchins in Brazil, take well over two years to learn to crack nuts (Inoue-Nakamura \& Matsuzawa, 1997; de Resende et al., 2008). By six years of age chimpanzees are still less efficient at ant-dipping than adults (Nishida \& Hiraiwa, 1982; Humle, 2006).

Laboratory studies indicate that the development of tool use in NC crows follows a similar pattern to that described above for primates. Kenward et al. $(2005,2006)$ describe the ontogeny of tool-oriented behaviours in NC crows as a combination of inherited action patterns, social and individual learning and creative problem solving. The authors hand-raised four NC crows in artificial nests and provided them with sticks and food that could only be extracted with tools. As is the case with primates, food retrieval was preceded by precursor actions that resembled components of proficient tool use. However, compared to the long learning periods reported for young primates, all four hand-raised juveniles retrieved food relatively early at around 70 days of age. Two of the four juvenile crows were allowed to watch tool use by their human foster parents, which resulted in increased twig carrying and insertion rates. However, the tutoring did not influence the onset or proficiency of food extraction, indicating that social input might not be necessary to acquire proficiency in basic stick tool use. The same is true for other bird species that habitually use tools in the wild. Woodpecker finches (Cactospiza pallida) showed similar pre-functional development of tool behaviour and acquired proficient stick tool use regardless of whether or not they had a tool-using model to learn from (Tebbich et al., 2001). Similarly, naïve Egyptian vultures (Neophron percnopterus) can develop the technique of throwing stones to break eggs without social input (Thouless et al., 1989). However, in the Kenward et al. (2006) study, the tutored crows also had a preference to handle objects that had been manipulated by the experimenters, indicating that stimulus enhancement might play a role in the acquisition of certain aspects of tool manufacture and use. Several weeks after developing stick tool use, the four crows were each presented with single, artificially mounted pandanus leaves. All the crows ripped at the leaves and removed strips of material. One 3-month-old individual made a strip and used it as a probe on its first day of exposure to the leaf (Kenward et al., 2005). Kenward et al. concluded 
that basic tool manufacture and use can develop from a disposition to manipulate tool-like material to try and obtain out-of-reach food, without the need for social learning.

Hunt et al.'s (2007) observations of a hand-raised male NC crow at Parc Zoo-Forestier, Nouméa confirmed that basic tool skills can develop without social learning. This crow developed stick tool use and tore off pieces of provided Pandanus sp. leaves, but he did not use them to extract meat. Similarly, when four captive adult crows that lacked experience with Pandanus leaves were given the opportunity to use and manufacture pandanus tools, only two of them used the provided tools and none manufactured tools. Hunt et al. (2007) proposed that a disposition for basic stick tool skills evolved early in the history of the NC crow's tool behaviour. With this disposition in place, crows then enhanced their stick tool skills and developed pandanus tool skills through individual and social learning. The fact that none of the pandanus tools manufactured by captive crows in Kenward et al.'s study resembled any of the three designs made in the wild is consistent with this hypothesis. Furthermore, the way these tools were manufactured did not resemble the distinct cutting and ripping techniques used by free-living adult crows to make pandanus tools (Hunt \& Gray, 2003, 2004b).

While research on hand-raised animals in the laboratory enables observations under controlled conditions, it does not necessarily model all processes that might lead to the development of complex behaviours in the wild. For example, the production of strips of pandanus leaf in the Kenward et al. (2005) study might be an artefact of impoverished living conditions in the laboratory. Adult crows held in our own outdoor aviary on the island of Maré sometimes also indiscriminately tear at pandanus leaves provided in their cages without using most of the leaf fragments as tools. Only a field study can reveal all the interactions between parents and offspring that might facilitate various forms of social learning. Investigating the development of tool manufacture and use in natural conditions is, therefore, crucial for exploring the mechanisms that actually underlie the transmission of complex tool skills. In this study we document the development of pandanus tool manufacture and use in wild NC crows. Over the course of two years we observed the tool development of six juvenile crows that visited feeding sites in their natural habitat. The study was carried out on the island of Maré, New Caledonia, where crows habitually manufacture wide pandanus tools and basic, non-hooked stick tools. 


\section{Methods}

\section{Study site and subjects}

The study was carried out on the island of Maré, New Caledonia, about $5 \mathrm{~km}$ inland from Wabao village. We observed crows at or close to feeding tables in the forest in most months in 2005 (January-May, July and OctoberDecember) and 2006 (January-May, August and October-December). One juvenile crow (Abel) was also subsequently observed in late 2008. NC crows breed on Maré during November and December and chicks have usually fledged by January.

We documented the behaviour of six juveniles that regularly visited the feeding tables. While other crows also occasionally visited the tables we only recorded data on target birds and their family members (parents and/or siblings, Table 1). Pandora's partner (and Primo's father; family 1) was found dead in November 2004 from a probable goshawk (Accipiter sp.) attack, before Primo hatched. Pandora then paired with Abraxas (family 2) around May 2005 and successfully raised Yor in 2006. Adam and Godot (family 3) successfully raised two siblings, Cain and Abel, in the 2005/2006 breeding season. Klaus and Maui (family 4) failed to raise chicks in 2003 and 2004, but successfully raised Bo in the 2005/2006 breeding season. Twiggy (family 5) was observed only in December 2005 and his parents were unknown. All study animals (except for one female parent, Pandora), were banded with individually coloured leg bands. Some birds had previously participated in experimental work in 2003/2004 at feeding tables (Pandora) or in our on-site aviary (Abraxas).

Primo was placed in the aviary for one week in January 2006 to record tool manufacture and meat extraction in order to increase sample size in the 10-12 month age class. For Cain, data on tool preference, tool manufacture and meat extraction at 28 months of age was also obtained in the aviary in April 2008, after he participated in experiments unrelated to this study.

\section{Procedure}

Observations were made at feeding tables at various times throughout the day. Feeding tables were around $1 \mathrm{~m}$ above the ground and made out of wood found in the vicinity. We positioned a fresh Pandanus sp. tree next to the table for pandanus tool manufacture, and usually a branch to encourage stick tool manufacture. On each table we placed a dead log in which we 
Table 1. Details of target juveniles and their families.

\begin{tabular}{lllcccc}
\hline & Crow & Status & Sex & Hatched & Fledged & Banding date \\
\hline Family 1 & Epi $^{\mathrm{a}}$ & Adult & $\sigma^{7}$ & - & - & $23 / 09 / 2003$ \\
& Pandora $^{7}$ & Adult & $\wp$ & - & - & Unbanded \\
& Primo & Juvenile & $\sigma^{7}$ & $02 / 12 / 2004^{\mathrm{b}}$ & $01 / 01 / 2005$ & $30 / 12 / 2004$ \\
Family 2 & Abraxas & Adult & $\sigma^{7}$ & - & - & $21 / 09 / 2003$ \\
& Pandora & Adult & $\wp$ & - & - & Unbanded \\
& Yor & Juvenile & $\sigma^{7}$ & $13 / 11 / 2005^{\mathrm{b}}$ & $14 / 12 / 2006$ & $13 / 12 / 2006$ \\
Family 3 & Adam & Adult & $\sigma^{7}$ & - & - & $23 / 07 / 2004$ \\
& Godot & Adult & $\wp$ & - & - & $24 / 07 / 2004$ \\
& Cain & Juvenile & $\sigma^{7}$ & $06 / 11 / 2005^{\mathrm{b}}$ & $06 / 12 / 2005^{\mathrm{b}}$ & $14 / 02 / 2006$ \\
& Abel & Juvenile & $\sigma^{7}$ & $06 / 11 / 2005^{\mathrm{b}}$ & $06 / 12 / 2005^{\mathrm{b}}$ & $14 / 02 / 2006$ \\
Family 4 & Klaus & Adult & $\sigma^{7}$ & - & - & $14 / 09 / 2003$ \\
& Maui & Adult & $\uparrow$ & - & - & $14 / 09 / 2003$ \\
& Bo & Juvenile & $\uparrow$ & $06 / 11 / 2005^{\mathrm{b}}$ & $06 / 12 / 2005^{\mathrm{b}}$ & $16 / 02 / 2006$ \\
Family 5 & $?$ & Adult & $\sigma^{7}$ & - & - & - \\
& $?$ & Adult & $\wp$ & - & - & - \\
& Twiggy & Juvenile & $\sigma^{7}$ & Dec $2004^{\mathrm{c}}$ & Jan $2005^{\mathrm{c}}$ & $04 / 08 / 2005$ \\
\hline
\end{tabular}

${ }^{a}$ Died in late 2004 before Primo fledged.

${ }^{\mathrm{b}}$ Date estimated from nest observations.

${ }^{\mathrm{c}}$ Date estimated from mouth colouring and behaviour at capture.

drilled vertical holes (6-7 $\mathrm{cm}$ deep and $2.5 \mathrm{~cm}$ in diameter). The holes were baited with pieces of meat that crows could only extract with tools. To create more natural feeding conditions, from April 2006 we usually also baited the Pandanus sp. tree at the table by pushing pieces of meat into crevices at the bases of the leaves. Over the course of the study we used eight feeding tables distributed in an area of ca. $0.4 \mathrm{~km}^{2}$. Observations at tables were videotaped from hides set up 6-10 m from tables. Video cameras were either handheld or operated automatically in conjunction with a motion detector (Wachit VMD-19M Video Motion Detector, Farco Technologies, New Zealand). During direct observation, the holes and the Pandanus sp. tree at the table were baited after each visit in which crows had extracted meat. At tables monitored automatically with a motion detector/camera, the holes and trees were baited several times a day.

Data analysis

Data extraction from video footage followed standard focal animal procedures (Altmann, 1974) and started whenever a focal juvenile landed on a 
feeding table. By observing video footage of one juvenile (Primo) we defined nine tool-related behaviours to describe the development of proficiency in pandanus tool manufacture and use (Table 2 and supplementary electronic material, Videos 1 and 2, see Appendix). Four of these nine behaviours describe tool use, and the remaining five tool manufacture (Techniques 0-4). The manufacture techniques range from unsuccessful, random-like ripping of leaves (Technique 0) to adult-like manufacture (Technique 4). For each visit by a target crow to a feeding table we recorded: (1) visit duration, (2) the family members that shared the table, (3) the number of tools manufactured and used, (4) the duration of each tool manufacture from the moment a crow touched the leaf with the bill to the removal of the tool from the leaf, (5) the amount of time probing for meat (probing was recorded only when a crow was actively using a tool in a hole), (6) the occurrence of tool-related behaviours according to Table 2, (7) whether a juvenile watched parental tool manufacture and meat extraction (see below for more details) and (8) parental feeding of a juvenile.

A juvenile was recorded as watching its parent only when the following four criteria were met: (1) the distance to the parent was less than ca. $1 \mathrm{~m}$, (2) the juvenile was either side-on to the parent or facing it, (3) the juvenile was not obviously engaged in another activity (e.g., eating or using a tool) and (4) the line of vision between juvenile and parent was not obstructed.

We measured the development of proficiency in pandanus tool manufacture over time in various ways. First, we separated the developmental period into four post-hatching age classes: 2-3 months, 4-6 months, 7-9 months and 10-12 months. We only began collecting data at the end of the second month post-hatching because juveniles only visited the feeding tables from that time onwards. We calculated the frequency that individuals in each age class used each of the five manufacture techniques (Table 2). To measure probing efficiency we first calculated the ratio of faulty probing time (first three tool use categories in Table 2 combined) to total probing time (whether successful or not) for each bird in each age class, then the mean ratio across individuals in the age classes. For meat extraction times we first calculated the mean extraction time per visit to a feeding table (total amount of successful probing time (s) divided by the number of extractions). We then calculated the mean extraction time across individuals in each age class. To determine the amount of unrewarded probing, we calculated the time spent 
Table 2. Ethogram definitions of tool related behaviours (also see supplementary electronic material, Videos 1 and 2).

\begin{tabular}{|c|c|c|c|}
\hline Category & Behaviour & Measured & Description \\
\hline Tool use & $\begin{array}{l}\text { Wrong-angle } \\
\text { probing* }\end{array}$ & d & $\begin{array}{l}\text { Holding a twig and performing probing mo- } \\
\text { tions directed towards a hole. Because the twig } \\
\text { is held at the wrong angle the hole is missed and } \\
\text { no insertion takes place. }\end{array}$ \\
\hline Tool use & Folding & $\mathrm{d}$ & $\begin{array}{l}\text { Grasping a tool that is already inserted in a hole } \\
\text { and bending or folding it in the hole. }\end{array}$ \\
\hline Tool use & $\begin{array}{l}\text { Defective } \\
\text { probing }\end{array}$ & $\mathrm{d}$ & $\begin{array}{l}\text { Probing with a tool that is inserted in a non- } \\
\text { functional way (e.g., bent, both ends pointing } \\
\text { upwards). }\end{array}$ \\
\hline Tool use & Probing & $\mathrm{d}$ & $\begin{array}{l}\text { Inserting tool into hole or crevice/inter-leaf } \\
\text { space, followed by up and down movements } \\
\text { with the head, holding the tool. }\end{array}$ \\
\hline $\begin{array}{l}\text { Tool } \\
\text { manufacture }\end{array}$ & Technique 0 & $\mathrm{~d}, \mathrm{n}$ & $\begin{array}{l}\text { Apparently random, not sequential ripping at } \\
\text { pandanus leaf that does not result in part of the } \\
\text { leaf coming off. }\end{array}$ \\
\hline $\begin{array}{l}\text { Tool } \\
\text { manufacture }\end{array}$ & Technique 1 & $\mathrm{~d}, \mathrm{n}$ & $\begin{array}{l}\text { Apparently random, not sequential ripping at } \\
\text { pandanus leaf resulting in part of the leaf com- } \\
\text { ing off and being used as a tool. }\end{array}$ \\
\hline $\begin{array}{l}\text { Tool } \\
\text { manufacture }\end{array}$ & Technique 2 & $\mathrm{~d}, \mathrm{n}$ & $\begin{array}{l}\text { Coordinated sequence of cutting and ripping at } \\
\text { a leaf edge. Because rips do not align, the crow } \\
\text { cannot remove the tool from the leaf. }\end{array}$ \\
\hline $\begin{array}{l}\text { Tool } \\
\text { manufacture }\end{array}$ & Technique 3 & $\mathrm{~d}, \mathrm{n}$ & $\begin{array}{l}\text { Coordinated sequence of cutting and ripping at } \\
\text { a leaf edge. Because the second cut/rip is made } \\
\text { proximal to the first, this results in a tool of } \\
\text { adult-like shape but with the barbs pointing to- } \\
\text { wards the working tip. }\end{array}$ \\
\hline $\begin{array}{l}\text { Tool } \\
\text { manufacture }\end{array}$ & Technique 4 & $\mathrm{~d}, \mathrm{n}$ & $\begin{array}{l}\text { Adult-like tool manufacture. Coordinated se- } \\
\text { quence of cutting and ripping at a leaf edge re- } \\
\text { sulting in a functional tool with the barbs point- } \\
\text { ing away from the working tip. }\end{array}$ \\
\hline
\end{tabular}

\footnotetext{
* Definition and description corresponds to that defined in Kenward et al. (2006). The tool use categories 'wrong-angle probing', 'folding' and 'defective probing' were combined into one category 'faulty probing' for analysis. $d=$ duration of activity measured in seconds; $n=$ frequency of behaviour or number of manufactured tools.
}

in unsuccessful probing events in relation to total probing time in each age class. 
Data were analysed using non-parametric statistics because they did not conform to assumptions for normality. Spearman's Rho was used to correlate age with the different developmental measures of tool manufacture and use. To calculate correlations we only used juveniles that provided data in at least three different age classes and developed a preference for pandanus tools (Primo, Yor and Cain). Data for these birds were combined. Yor rarely extracted meat from the provided logs; therefore, we could only analyze the tool use of Primo and Cain. All correlations were one-tailed. We used $\chi^{2}$ tests to compare the following behaviour between individual adult and juvenile pairings: the frequency of tool manufacture techniques, unrewarded probing and tool manufacture variants. To determine whether a crow had a dominant tool manufacture variant we tested the frequency of the most used variant against the combined frequencies of the two variants the crow used least. Binomial tests were used to identify individual preferences for either stick or pandanus tools. The $\chi^{2}$ and binomial tests were two-tailed. MannWhitney $U$-tests were used to compare extraction times and the duration of tool manufacture between individual adult and juvenile pairings. The $\alpha$ level was 0.05 for all tests.

\section{Results}

During ca. $1790 \mathrm{~h}$ of direct observation and $2000 \mathrm{~h}$ of observation with motion detector units we video-taped ca. $30 \mathrm{~h}$ of juvenile crow behaviour. We recorded 716 visits to feeding tables by the six target juveniles. Their visits lasted on average $4.1 \mathrm{~min}$ (range $4 \mathrm{~s}$ to $22.1 \mathrm{~min}$ ). We recorded the manufacture of 615 pandanus tools by the six juveniles and 421 tools by their parents. We observed the juveniles extract 521 pieces of meat with pandanus tools from holes and Pandanus sp. trees and their parents extract 469 pieces of meat.

\section{Opportunity to watch parental tool manufacture and use}

Four juveniles (Primo, Yor, Cain and Abel) regularly visited feeding tables with at least one pandanus-tool-using parent, sharing a table with the parent for $30-40 \%$ of the visiting time up to six months of age (Figure 1). The parents manufactured pandanus tools at an average rate of 0.01 tools per min of visit time. The proportion of time spent with their parents declined 


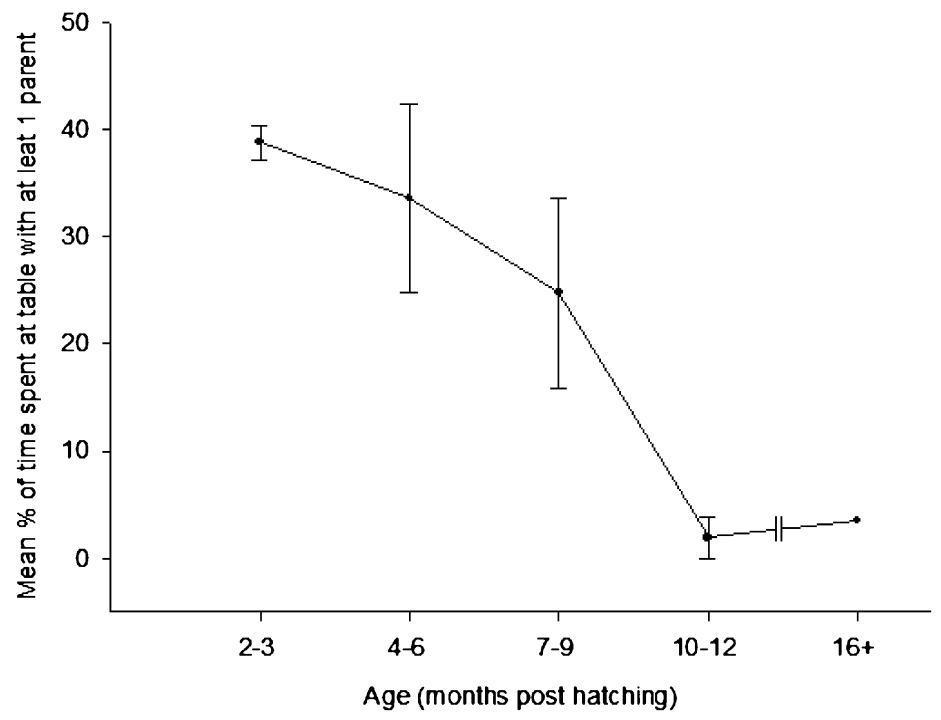

Figure 1. Mean $\%$ of time spent with parent(s) at feeding table.

with age (Spearman's $r=-0.73, N=12, p<0.004$ ). In each age class, juveniles watched parents extract meat from holes and trees more frequently than they watched pandanus tool manufacture. This difference was only nonsignificant at 7-9 months of age (Figure 2, 2-3 months: $\chi^{2}=7.7, \mathrm{df}=1$, $p=0.006$; 4-6 months: $\chi^{2}=104.2, \mathrm{df}=1, p<0.001$; 7-9 months: $\chi^{2}=3.1, \mathrm{df}=1, p=0.08,10-12$ months: $\chi^{2}=5.0, \mathrm{df}=1, p=0.03$ ). Juveniles often approached a parent very closely when it was extracting meat with a tool, sometimes looking into the hole that the parent was extracting meat from and even touching the tool that the parent was using. This was not the case with juveniles watching tool manufacture, which was mostly from some distance away.

\section{Development of pandanus tool manufacture}

\section{Development of tool manufacture techniques}

Pandanus tool manufacture developed similarly in Primo, Yor and Cain (Figure 3). Techniques 0 and 1 (uncoordinated ripping at pandanus leaves that sometimes resulted in a tool, see Video 2 for tool manufacture techniques) were used mostly up to six months of age. The relative frequency of this behaviour declined with age (data combined for Primo, Yor and Cain, Tech- 


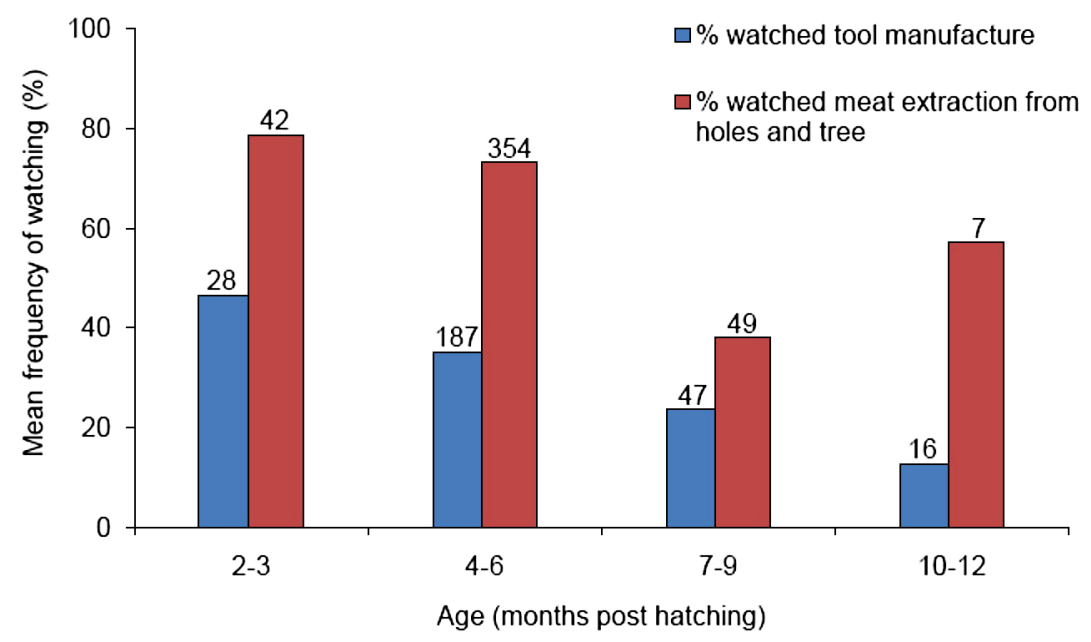

Figure 2. Juveniles watching tool manufacture and meat extraction. The numbers on top of the bars represent the total numbers of parental tool manufactures and meat extractions when a juvenile was present. Only Cain contributed to the 10-12 months age class. This figure is published in colour in the online edition of this journal, that can be accessed via http://www.brill.nl/beh

nique 0: Spearman's $r=-0.95, N=10, p<0.001$, Technique 1: Spearman's $r=-0.55, N=10, p=0.049$ ). From about four months of age juveniles employed more coordinated sequences of ripping and cutting at leaf edges. Because these rips did not align (Technique 2) or were performed in the wrong order (Technique 3), the tools either could not be removed from the leaf or were oriented with the barbs pointing downwards and, therefore, were not immediately functional. The frequency of Techniques 2 and 3 was low and constant across age classes after 3 months of age. Adult-like tool manufacture (Technique 4: coordinated sequence of cutting and ripping that produced a correctly oriented, functional tool) appeared first at 4-6 months of age, and its frequency increased over time (data combined for Primo, Yor and Cain: Spearman's $r=-0.77, N=10, p<0.005$ ).

However, at 10-12 months of age the relative frequency of adult-like tool manufacture was still significantly lower than that of their respective parents for two of the three juveniles (Figure 3; Primo: $\chi^{2}=21.9, \mathrm{df}=1$, $p<0.001$; Cain: $\left.\chi^{2}=22.9, \mathrm{df}=1, p<0.001\right)$. Yor was making pandanus tools in adult-like fashion and frequency at 7-9 months of age $\left(\chi^{2}=0.1\right.$, $\mathrm{df}=1, p=0.74)$. At 16 and 28 months of age Primo and Cain, respectively, had adult-like frequencies of manufacture techniques (Primo: $\chi^{2}=3.6, \mathrm{df}=$ 
Cain

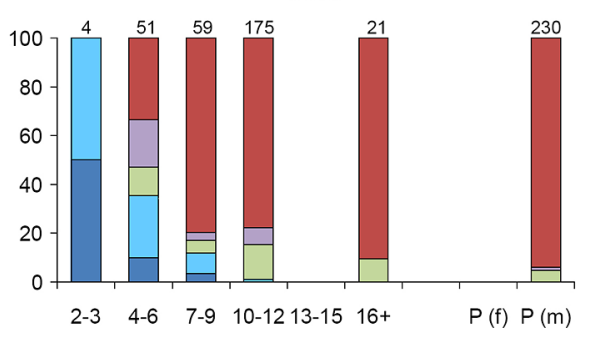

Primo

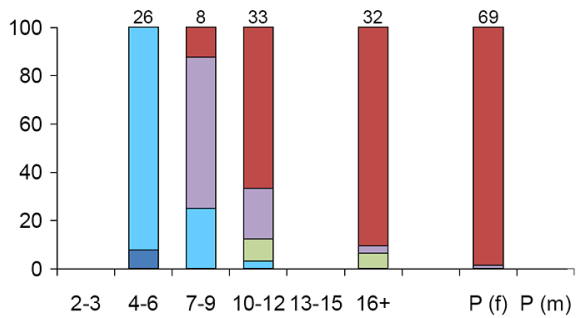

Bo

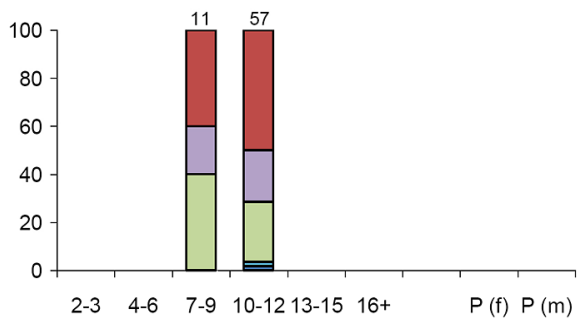

Abel

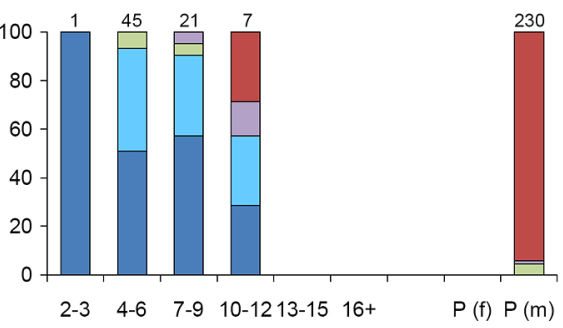

Yor

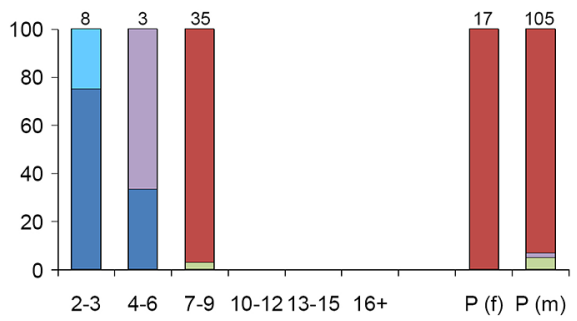

Twiggy

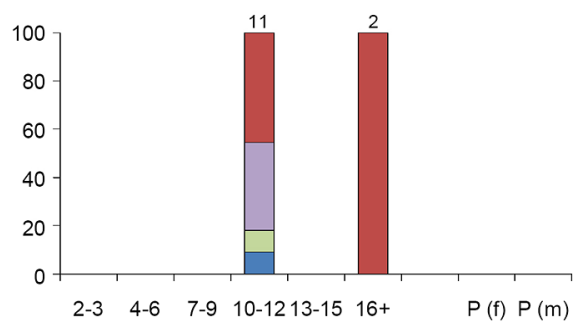

Figure 3. Individual development of pandanus tool manufacture techniques. ( $\square$ ) Technique 0 (random rips, no tool); ( $\square$ ) Technique 1 (random rips, tool removed); ( $\square$ ) Technique 2 (coordinated, non-aligning rips, no tool removed); ( $\square$ ) Technique 3 (coordinated rips in wrong order, barbs towards working tip); ( $\square$ ) Technique 4 (adult-like tool manufacture). $\mathrm{P}=$ parent $\mathrm{f}=$ female $\mathrm{m}=$ male, $x$-axis $=$ age (months post hatching), $y$-axis = frequency of manufacture technique (\%). The numbers on top of the bars represent the total number of tools manufactured in each age class. This figure is published in colour in the online edition of this journal, that can be accessed via http://www.brill.nl/beh

1, $p=0.09$; Cain: $\left.\chi^{2}=0.4, \mathrm{df}=1, p=0.54\right)$. We observed no parental tool manufacture for juveniles Bo and Twiggy. There was insufficient data to statistically correlate age with manufacture techniques for Bo and Twiggy, but the relative frequencies of techniques used by them fit well the pattern shown by Primo, Yor and Cain (Figure 3). Abel's performance differed considerably from the other juveniles in that adult-like tool manufacture only 
Table 3. Preference for use and manufacture of pandanus or stick tools at tables in each age class.

\begin{tabular}{lcccccccc}
\hline Bird & Tool & $2-3$ & $4-6$ & $7-9$ & $10-12$ & $16+$ & Parent $\left(\sigma^{\top}\right)$ & Parent (o) \\
\hline Primo & $\mathrm{p}$ & $11 / 1^{*}$ & $22 / 24$ & $1 / 8$ & $2 / 33^{*}$ & $6 / 34^{*}$ & & $0 / 69^{*}$ \\
& $\mathrm{~s}$ & $0 / 3$ & $17 / 13$ & $1 / 3$ & $3 / 0$ & $0 / 0$ & & $0 / 0$ \\
Yor & $\mathrm{p}$ & $6 / 8^{*}$ & $4 / 3$ & $21 / 39^{*}$ & & & $0 / 105^{*}$ & $0 / 17^{*}$ \\
& $\mathrm{~s}$ & $1 / 0$ & $2 / 0$ & $0 / 0$ & & & $19 / 0$ & $0 / 0$ \\
Cain & $\mathrm{p}$ & $1 / 4$ & $50 / 51^{*}$ & $9 / 59^{*}$ & $8 / 175^{*}$ & $16 / 21^{*}$ & $0 / 230^{*}$ & $?$ \\
& $\mathrm{~s}$ & $1 / 0$ & $2 / 2$ & $2 / 0$ & $5 / 0$ & $8 / 0$ & $0 / 0$ & $?$ \\
\multirow{4}{*}{ Abel } & $\mathrm{p}$ & $4 / 1$ & $76 / 45^{*}$ & $34 / 21$ & $31 / 7$ & $2 / 3$ & $0 / 230^{*}$ & $?$ \\
& $\mathrm{~s}$ & $1 / 0$ & $48 / 11$ & $42 / 6$ & $90 / 3^{*}$ & $14 / 1^{*}$ & $0 / 0$ & $?$ \\
Bo & $\mathrm{p}$ & & & $1 / 11^{*}$ & $2 / 57^{*}$ & & $0 / 0$ & $\left(22 / 22^{*}\right)$ \\
& $\mathrm{s}$ & & & $0 / 0$ & $9 / 0$ & & $42 / 8^{*}$ & $(0 / 0)$ \\
\multirow{2}{*}{ Twiggy } & $\mathrm{p}$ & & & & $0 / 11^{*}$ & $0 / 2$ & $?$ & $?$ \\
& $\mathrm{~s}$ & & & & $0 / 0$ & $0 / 0$ & $?$ & $?$ \\
\hline
\end{tabular}

The first number indicates tools picked up on, or brought to, the table for use, and the second number indicates tools manufactured at the table. $\mathrm{p}=$ pandanus, $\mathrm{s}=$ stick. Asterisk indicates a significant preference for either stick or pandanus tools (picked up + manufactured tools, binomial test, $p<0.05$ ). For Bo's mother, tool preference had been established in 2004. Data for Abel at over 16 months is based on observations in 2008 when he was between 2 and 3 years old.

appeared twice at 12 months of age. Because he was the only juvenile that preferred to pick up stick tools rather than manufacture pandanus tools (Table 3) we excluded him from all analyses concerning pandanus tool manufacture and use.

The shape of a pandanus tool generally reflects the technique that it was manufactured with (Figure 4). Technique 0 and 2 tools cannot be removed from the leaf and remain on the pandanus tree. Technique 1 tools do not closely resemble the shape of a classic wide pandanus tool (uniformly wide with barbs along one edge). Technique 3 tools were adult-like in shape but not immediately functional because the barbs point towards the working tip of the tool. Technique 4 tools cannot be distinguished from adult tools.

\section{Duration of tool manufacture}

Manufacture Technique 4 appears to indicate adult-like technical proficiency in producing wide tools. However, juveniles using Technique 4 took longer 

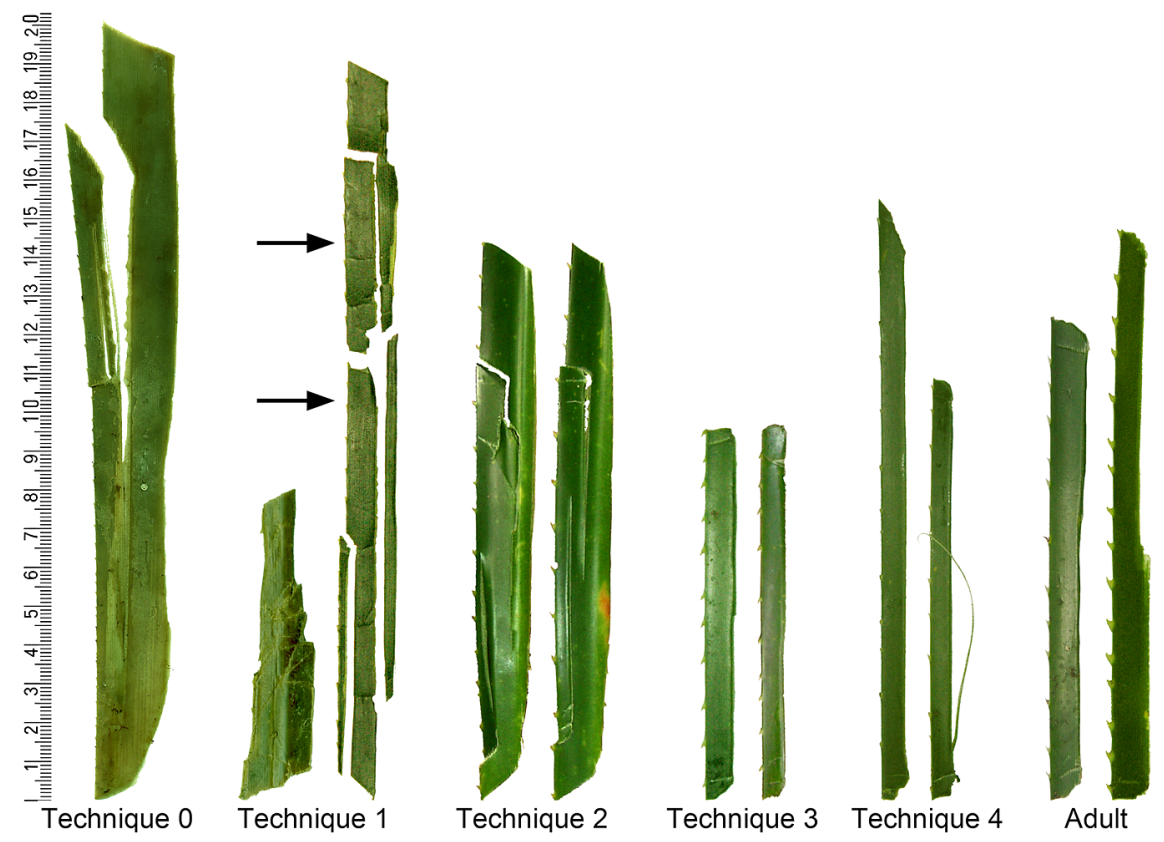

Figure 4. Examples of tools manufactured with Techniques 0-4 compared with adult tools. Techniques 0 and 2 do not result in a tool being removed from the leaf. We, therefore, show the section of the leaf where the tool manufacture had been attempted. The right hand tool of Technique 1 is the result of multiple uncoordinated ripping at the same leaf; only the two pieces indicated by arrows were used to probe in a hole. Techniques 3 and 4 produce adultlike tools, but the tools in Technique 3 are removed from the leaf in the wrong orientation. The stringy edge of the right hand tool for Technique 4 is caused by rips that do not align perfectly. This figure is published in colour in the online edition of this journal, that can be accessed via http://www.brill.nl/beh

to manufacture tools than their parents, and at 10-12 months still had not reached the speed of their respective parent's manufacture (Figure 5; Primo/Pandora: $U=204.5, N_{1}=22, N_{2}=68, p<0.001$; Yor/Pandora + Abraxas: $U=566, N_{1}=37, N_{2}=93, p<0.001$; Cain/Adam: $U=$ 6929, $\left.N_{1}=136, N_{2}=160, p<0.001\right)$. The duration of juveniles' tool manufacture is negatively correlated with age, indicating an increase in manufacture speed over time (Spearman's $r=-0.13, N=260, p=0.016$ ). By 16 months of age Primo had reached the manufacture speed of his mother ( $U=827, N_{1}=31, N_{2}=68, p=0.08$ ), while Cain at 28 months of age still took significantly longer than his father to manufacture wide pandanus tools $\left(U=389, N_{1}=12, N_{2}=160, p<0.001\right)$. 


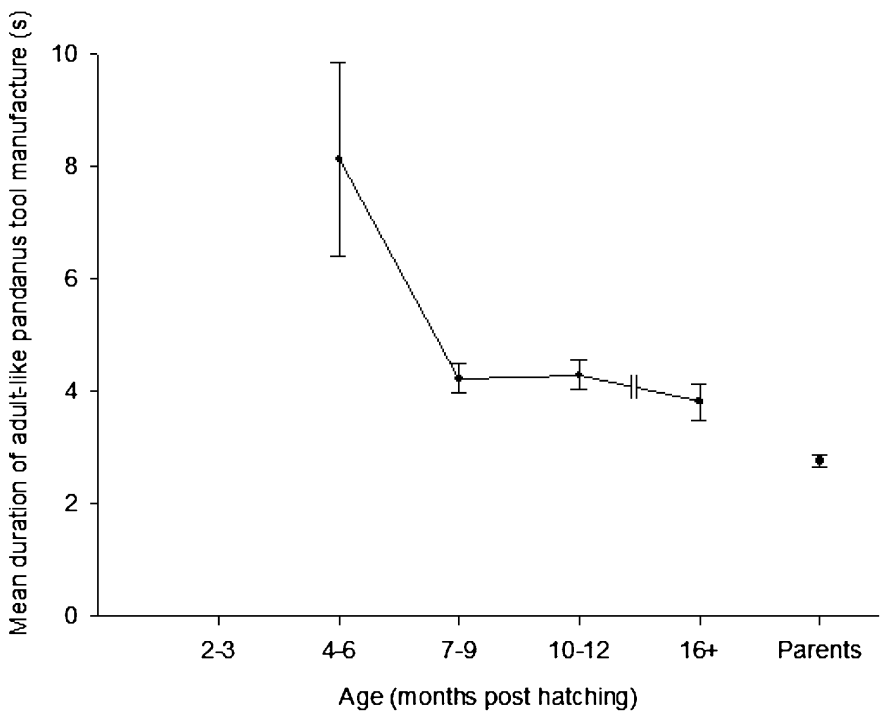

Figure 5. Duration of adult-like tool manufacture.

\section{Variants of tool manufacture}

Close examination of manufacture techniques revealed three different variants of wide tool manufacture (Figure 6, Video 3). In Variant A (cut-rip and cut-rip), two cut-rip sequences converge about half way along the tool. In Variant B (cut-rip and cut), the first cut is followed by a long rip (action 1). The tool is then removed from the main leaf with only a cut (action 2). In Variant C (cut and cut-rip) the actions in Variant B are reversed: only a cut (action 1) is made initially, then the tool is removed by a cut-rip sequence (action 2). All variants may also occur when birds use manufacture Techniques 2 (rips do not align; therefore, the tool cannot be removed from the leaf) and 3 (second cut/rip starts proximal to the first cut/rip; therefore, the tool is removed with the barbs pointing towards the working tip). Figure 7 shows the frequencies that families 1-3 used these variants (juveniles in these families often visited tables with at least one parent). Abraxas was the only bird in these families that did not have a significant preference for a particular variant. While Pandora clearly preferred Variant $\mathrm{B}\left(\chi^{2}=27.6, \mathrm{df}=\right.$ $1, p<0.001$ ), both her juveniles (Primo and Yor) predominantly manufactured tools by Variant A (Primo: $\chi^{2}=24$, df $=1, p<0.001$; Yor: $\chi^{2}=7.1$, $\mathrm{df}=1, p<0.011)$. Yor and Primo used Variant A significantly more often than any individual in this family (all $\chi^{2}>10.2$, all $\mathrm{df}=1$, all $p<0.002$ ). 


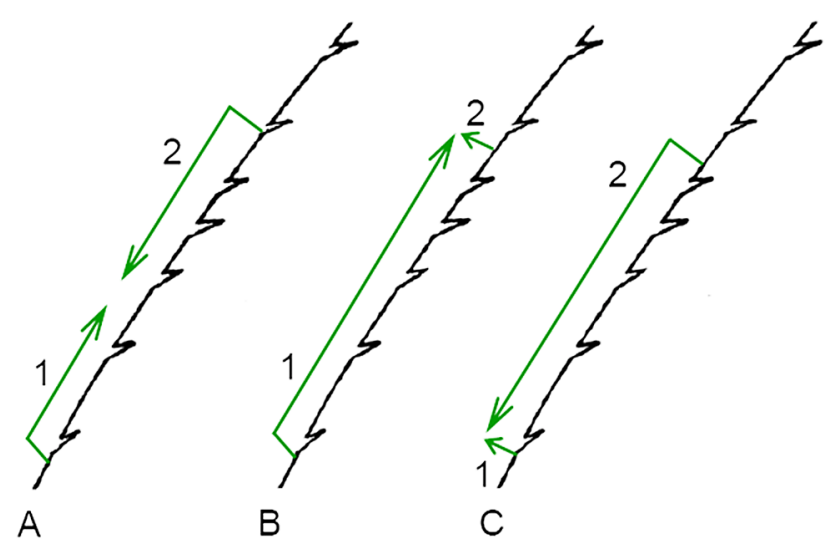

Figure 6. Variants of tool manufacture. Variant A: two cut-rip sequences converge about half way along the tool. Variant B: a cut-rip (1) is followed by a cut (2). Variant C: A cut (1) is followed by a cut-rip (2). This figure is published in colour in the online edition of this journal, that can be accessed via http://www.brill.nl/beh

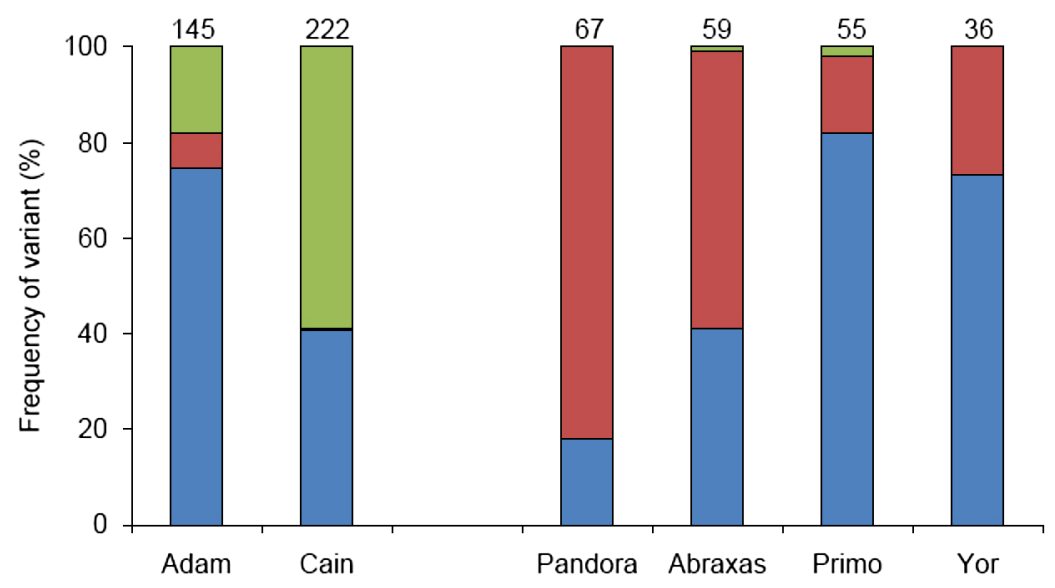

Figure 7. Variants of adult-like tool manufacture. Sample sizes are on top of bars. This figure is published in colour in the online edition of this journal, that can be accessed via http://www.brill.nl/beh. $\square$ Variation A; $\square$ Variation B; $\square$ Variation C.

In family 3, Adam preferred to use Variant A while his juvenile Cain was the only crow with a preference for Variant $\mathrm{C}$ (Adam: $\chi^{2}=34.8 \mathrm{df}=1$, $p<0.001$; Cain: $\left.\chi^{2}=7.1, \mathrm{df}=1, p=0.009\right)$. However, both Caine and Adam used Variant $\mathrm{C}$ significantly more often than any crow in Pandora's families ( $\chi^{2}$ tests for Cain and Adam vs. each member of Pandora's family: all $\chi^{2}>9.85$, all $\mathrm{df}=1$, all $p<0.002$ ). 
Starting pandanus tool manufacture at damaged leaf edge

The process of pandanus tool manufacture means that the missing section of leaf edge resulting from manufacture is an exact template, or counterpart, of the shape of the removed tool (Hunt, 2000a). While adult crows usually start tool manufacture at an intact leaf edge, juveniles frequently began tool manufacture at counterparts or other damaged parts of a leaf such as unfinished tools or broken edges (Figure 8). The proportion of tool manufactures starting at intact leaves increased with age (Spearman's $r=1, N=4, p<0.001)$. At $10-12$ months of age Cain rarely began tool manufacture where there was leaf damage, while at 16 months of age Primo still did so more than his mother Pandora (Cain: $\chi^{2}=3.4, \mathrm{df}=1, p=0.06$; Primo: $\chi^{2}=6.9, \mathrm{df}=1, p=0.009$ ).

\section{Development of tool preference}

Adult NC crows at our study site usually have a distinct preference to manufacture and use either stick tools or pandanus tools (Hunt \& Gray, 2007). All juveniles had at least one parent that preferred pandanus tools. Juveniles tended to use and/or manufacture both stick tools and pandanus tools during the first six months post hatching. By 10-12 months of age all juveniles

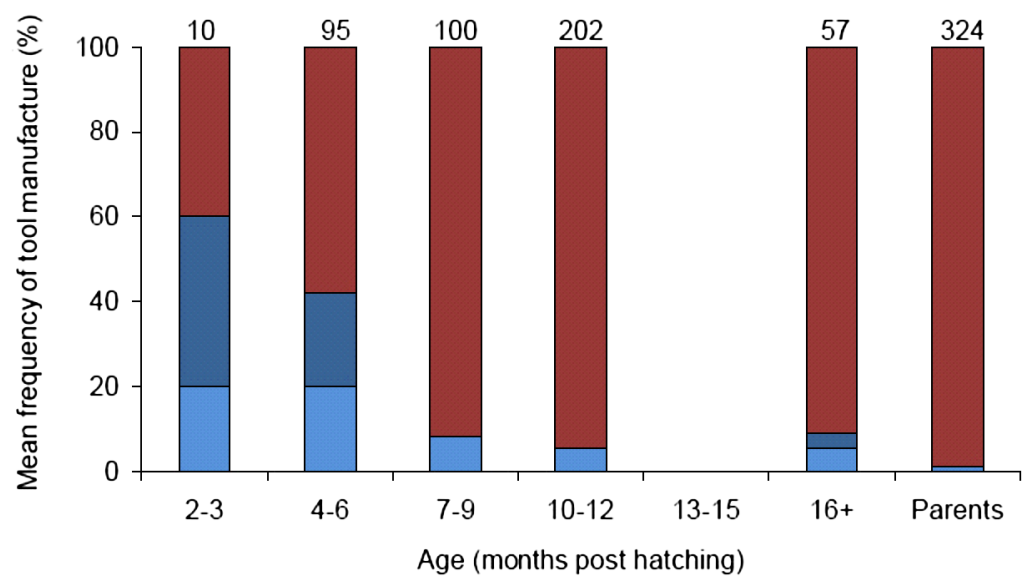

Figure 8. Average frequency of pandanus tool manufacture starting at counterparts or other damaged parts of the leaf. Numbers on top of bars represent the total number of tool manufactures per age class. This figure is published in colour in the online edition of this journal, that can be accessed via http://www.brill.nl/beh. $\square$ At CP; $\square$ at other damaged parts of the leaf; $\square$ at intact leaf. 
except Abel had developed a preference for pandanus tool manufacture (Table 3). When using tools they had not manufactured themselves, the birds discriminated less between a stick and a pandanus tool. However, at 10-12 months of age the juveniles' use of tools that they had not made was minimal. The tool preferences appeared to continue into adulthood: Primo and Cain still preferred pandanus tools at 16 and 28 months of age, respectively. Observations of Abel at ca. 3 years of age suggested that he still preferred stick tools to pandanus tools (Table 3 ).

\section{Development of pandanus tool use}

Origin of pandanus tools used at feeding tables

Up to 6 months of age the juveniles mainly used tools that had been left at the table by other crows (Figure 9). Many of these tools (38 of 94) had been used by a parent immediately before the juvenile picked it up. The frequency of use of parents' tools might be higher than we documented because some of the tools of unknown origin that juveniles picked up may have also been made by a parent. From seven months of age onwards, juveniles predominantly used tools they had manufactured themselves (Figure 9, Spearman's $r=-0.93, N=11, p<0.001)$.

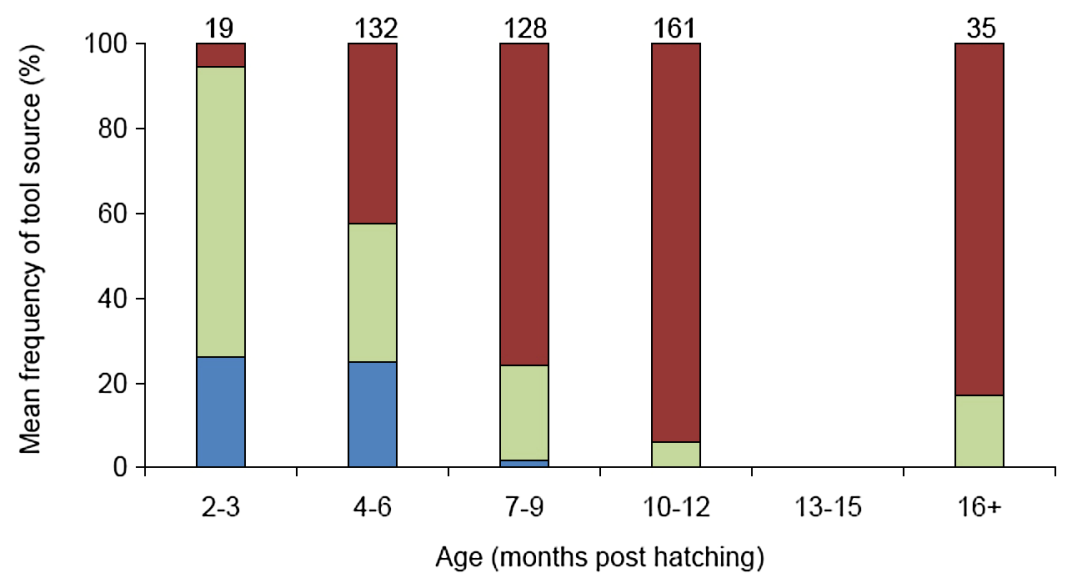

Figure 9. Origin of pandanus tools used at tables. Numbers on top of bars represent the total number of tools per age class. This figure is published in colour in the online edition of this journal, that can be accessed via http://www.brill.nl/beh. $\square$ Parent's tools; $\square$ other tools; 


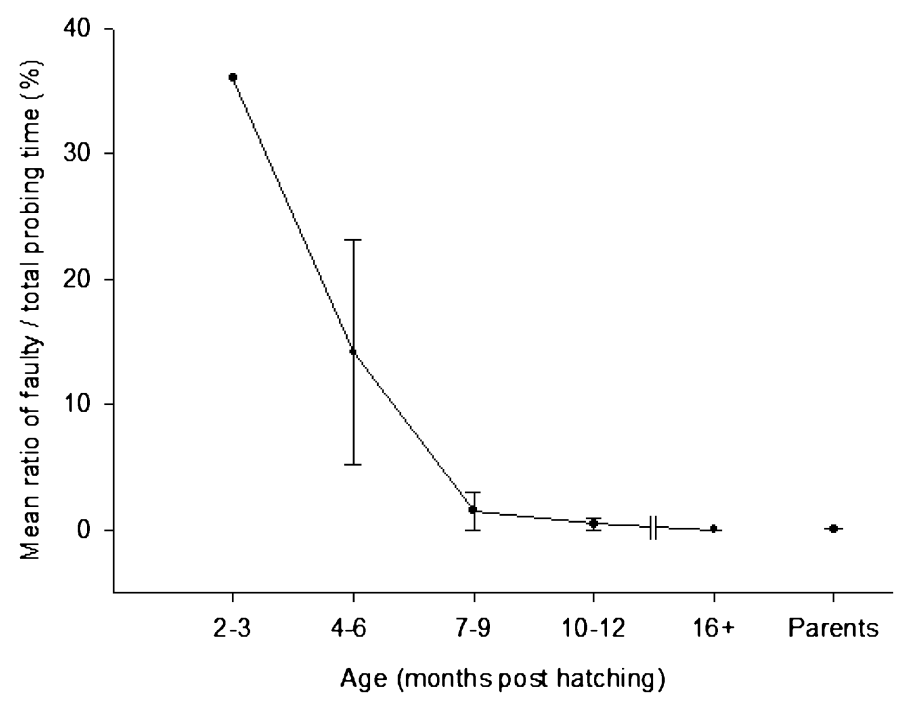

Figure 10. Mean ratio of faulty probing time to total probing time. Only Primo contributed to the $2-3$ months age class. Error bars represent $1 \mathrm{SE}$.

Development of proficient pandanus tool probing

Juveniles made three main mistakes when trying to extract meat from a hole with a pandanus tool: (1) wrong-angle probing, (2) folding a tool in a hole and (3) defective probing (Table 2). We combined these three behaviours into one category, 'faulty probing', because wrong-angle probing and folding rarely occurred. Faulty probing declined with age (Figure 10, Spearman's $r=-0.87, N=7, p<0.005$ ); after 6 months of age it accounted for less than $2 \%$ of the total probing time.

\section{Rewarded probing}

The time it took juveniles to successfully extract pieces of meat from holes declined over time (Figure 11; Spearman's $r=-0.43, N=69, p<0.001$ ). However, at 10-12 months of age both Primo and Cain still took significantly longer to extract meat than their respective parents (Primo/Pandora: $U=71.5, N_{1}=11, N_{2}=44, p<0.001$; Cain/Adam: $U=429, N_{1}=$ $\left.40, N_{2}=39, p<0.001\right)$. Primo's and Cain's extraction times did not differ significantly from their parents' at 16 and 28 months, respectively (Primo/Pandora: $U=95.5, N_{1}=5, N_{2}=44, p=0.63$; Cain/Adam: $\left.U=364.5, N_{1}=23, N_{2}=39, p=0.11\right)$. 


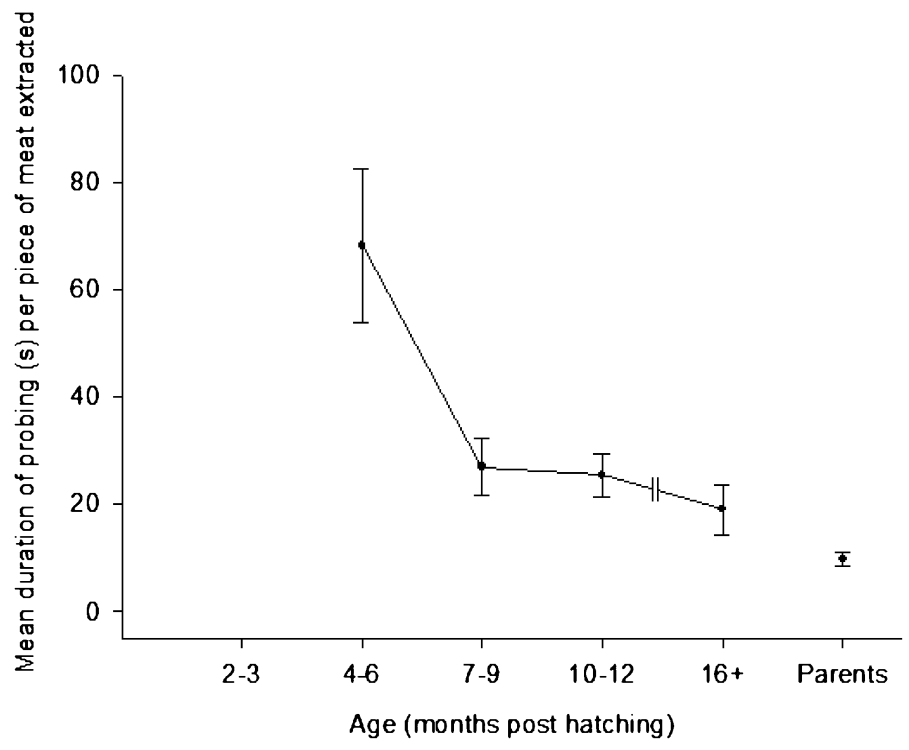

Figure 11. Mean duration of rewarded probing per piece of extracted meat from the holes in logs. Both Primo and Cain had reached adult-like speed at meat extraction at 18 and 28 months, respectively. Error bars represent 1 SE.

\section{Unrewarded probing}

A characteristic of juveniles' pandanus tool use was their persistence in probing without being rewarded. Up to 6 months of age they spent over 50\% of total probing time in unrewarded probing episodes (Figure 12). Although this ratio declined with age (Spearman's $r=-0.7, N=7, p=0.045$ ), juveniles still spent more time in unrewarded probing episodes at over 16 months of age than their respective parents (Primo over 16 months compared to Pandora: $\chi^{2}=48.9, \mathrm{df}=1, p<0.001$; Cain at 28 months compared to Adam: $\chi^{2}=206.6, \mathrm{df}=1, p<0.001$ ).

\section{Parental feeding}

Although juveniles up to 6 months of age spend a considerable amount of time on unsuccessful probing episodes (Figure 12), they were frequently fed meat that their parents extracted (Table 4). For example, when Cain was 4-6 months of age his father Adam extracted 192 pieces of meat at feeding tables and subsequently fed meat to Cain 103 times. Because a juvenile may be fed 


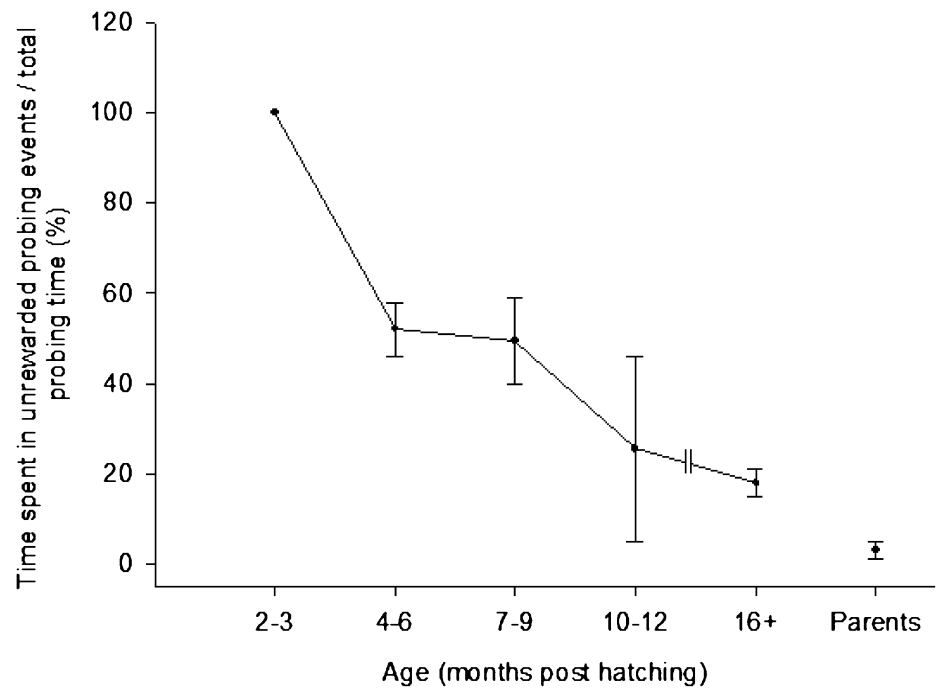

Figure 12. Mean ratio of unrewarded probing time to total probing time. Only Primo contributed to the 2-3 months age class. Error bars represent $1 \mathrm{SE}$.

Table 4. Parental feeding at tables in each age class.

\begin{tabular}{lccccc}
\hline Bird & $2-3$ & $4-6$ & $7-9$ & $10-12$ & $16+$ \\
\hline Primo & $20 / 6$ & $63 / 35$ & $0 / 0$ & $0 / 0$ & $0 / 0$ \\
Yor & $19 / 3$ & $10 / 2$ & $25 / 2$ & & \\
Cain & $2 / 1$ & $192 / 103$ & $25 / 0$ & $4 / 0$ & \\
Abel & $3 / 0$ & $222 / 79$ & $21 / 5$ & $2 / 0$ & \\
Bo & $14 / 2$ & $6 / 2$ & $5 / 0$ & $11 / 0$ & \\
\hline
\end{tabular}

The first number gives the total number of meat pieces extracted by parents from holes and pandanus trees in the presence of the juvenile. The second number gives the number of times parents subsequently fed the juvenile.

more than one piece of meat at a time Cain may have received more than 103 pieces.

\section{Discussion}

The results of our field study contrast with the rapid development of basic tool use observed in four hand-raised crows by Kenward et al. $(2005,2006)$. 
We found that the development of even the most basic form of pandanus tool manufacture (i.e., wide tools) is an extended process where juveniles reach adult proficiency only after a year or longer post-hatching. Our results are consistent, though, with the lengthy ontogeny of tool use in primates under natural conditions. For example, before human children master the complex task of using a spoon they perform repetitive actions like putting the spoon in the dish and removing it again (Connolly \& Dalgleish, 1989). This is followed by more complicated action sequences (e.g., putting the spoon in the dish, then in the mouth). Later on they incorporate functional actions (filling the spoon with food and eating) and eventually they include correction loops like checking if food is on the spoon before they put it in the mouth. Both chimpanzees and capuchins start learning about nut cracking by exploring surfaces, carrying out simple pounding actions of both nuts and stones and placing objects on surfaces (Visalberghi, 1987; Inoue-Nakamura \& Matsuzawa, 1997; de Resende et al., 2008). Such behaviour occurs before they correctly assemble all the physical elements of nut cracking and begin to open nuts successfully after two to three years of practice. Chimpanzees do not reach adult-like proficiency until they are nine to ten years old (Matsuzawa, 1994). Captive hand raised NC crows had predictable precursor actions that resembled aspects of experienced tool use but were not directly functional, such as rubbing a stick against a substrate and wrongangle probing. These precursor actions preceded successful food extraction and reached their maximum frequency at around eight weeks of age (Kenward et al., 2006). The reluctance of very young crows to come down to feeding tables meant that our earliest observations began only when the juveniles were eight weeks of age. This may explain why we rarely observed the precursor actions described by Kenward et al. (2006). However, we did find that juveniles went through four distinct subsequent stages in the development of proficient wide pandanus tool manufacture (Figures 3 and 4). At Stage I the first attempts to manufacture pandanus tools consisted of uncoordinated ripping of Pandanus sp. leaves, which often failed to produce a tool. If crows successfully removed the tool it often did not resemble the classic shape of an adult-made wide tool, or lacked barbs because it was removed at unsuitable locations for manufacture. At Stage II the crows only gradually produced adult-like wide tools with a well-coordinated sequence of cutting and ripping actions. Their use of adult-like cutting and ripping actions still did not always result in the removal of a functional tool from the leaf because 
they made errors in the position of a cut or a rip. Adults generally position the second cut/rip action distal to the first one, and both cuts have the same depth. They can, therefore, remove the tool easily from the leaf and hold it in a functional orientation (i.e., with the leaf-edge barbs facing away from the working end). In contrast, juveniles sometimes place the second cut/rip proximal to the first one. This results in the tool being held with the barbs facing towards the working end, which renders it non-functional. Furthermore, cut/rip actions may be of uneven depth and the tool cannot be removed from the leaf (Figures 3 and 4). At Stage III juveniles reached adult-like technical skill in wide tool manufacture, carrying out the correct sequence of manufacture steps. Complete adult-like proficiency, however, was only reached at Stage IV in the second year of life when juveniles' speed of manufacture matched that of adults.

Successful tool use was also preceded by a period of ineffective probing that included failure to insert the tool into the hole or probing with a tool that was incorrectly inserted (Video 1). Even when probing with a correctly inserted and oriented tool juveniles mostly failed to extract meat during the first 6 months post-hatching. Juveniles' persistence at unsuccessful tool use might be an important factor in enabling animals to acquire complex behaviours. Kenward et al. (2006) found that their four hand-raised crows performed precursor actions and inserted objects into holes and crevices for several weeks before they managed to extract meat. They suggest that this behaviour might be the result of an inherited tendency to find certain actions rewarding even if they were not associated with food. Unrewarded persistence with tool use has also been observed in other animals. For example, neither chimpanzees nor capuchins appear to get reinforced other than by scrounging or tolerated theft before they crack their first nut after several years of interacting and practicing with the materials (Biro et al., 2006; de Resende et al., 2008). In contrast, young crows frequently get fed a large part of the meat that they had just watched a parent extract. The parental feeding might help explain why juveniles persist with unrewarded tool manufacture and use for many months until they begin extracting their own prey.

The extensive trial and error learning that we observed by the juvenile crows is also seen in the development of tool use in other species. Tebbich et al. (2001) suggested that woodpecker finches (Cactospiza pallida) acquired tool use through a specific learning disposition that involves trial and error during a sensitive phase, without the need for social learning or reinforcement by obtaining food. Chimpanzees and capuchins also make a range 
of mistakes before successfully cracking their first nut (Inoue-Nakamura \& Matsuzawa, 1997; Hayashi et al., 2005; de Resende et al., 2008). According to action-perception theory (Lockman, 2000), these mistakes are important to enable individuals to learn about the properties of objects and their causal relations. Young NC crows probably need to learn some kind of basic physical knowledge about pandanus tools and probe sites. Early uncoordinated ripping of Pandanus sp. leaves might enable them to learn about their physical properties and that they have strong parallel fibers which facilitates the production of uniformly wide tools. By bending and folding pandanus tools into holes they experience that they are flat, flexible and only work when one end is inserted into the hole. Experimental work has shown that most adult $\mathrm{NC}$ crows do not pay attention to the presence and direction of barbs on wide pandanus tools (Holzhaider et al., 2008). Instead, they appear to obtain Stage III development above by associative learning about the correct sequence of manufacture actions required for successful food extraction.

An evolved disposition for certain action patterns (Kenward et al., 2005, 2006) combined with trial and error learning (Tebbich et al., 2001) appear to be sufficient to produce proficient stick tool use in NC crows and woodpecker finches. However, it is unlikely that such mechanisms alone can explain the complex tool skills seen in free-living crows. Our results are consistent with the view that various forms of social learning play an important role in maintaining complex tool skills in NC crows (Hunt \& Gray, 2003; Hunt et al., 2007). They show that juveniles have ample opportunity to learn socially via simple mechanisms such as local and stimulus enhancement. Juveniles visited tables together with one or both parents throughout their first year of life, but most commonly up to six months of age (Figure 1). While adult crows at tables generally manufactured their own pandanus tools to extract meat, juveniles up to six months of age predominantly used tools that were discarded or placed on the table after use by an adult (Figure 9). Many of these tools had been used by a parent immediately before the juvenile picked it up. This confirms Kenward et al.'s (2006) finding that juveniles had a preference for objects that had been manipulated by a human demonstrator. We found no evidence of active teaching such as error correction by parent crows, but their tolerance to juveniles taking and using their tools is likely to have facilitated learning by the juveniles. Juvenile chimpanzees also frequently use tools that have been left by their mothers or other members of the community (Matsuzawa et al., 2001; Biro et al., 2006). Laboratory studies confirm that 
naïve subjects profit from using tools formerly used or manufactured by an experienced individual (Hirata \& Morimura, 2000; Hirata \& Celli, 2003). On Maré, we often find pandanus tools left at the bases of Pandanus sp. leaves away from feeding tables where juvenile crows could easily find them.

Adult-made pandanus tools provide juveniles with opportunities to use suitable material for their early learning about tool use. Similarly, the presence of counterparts on leaves appear to facilitate juvenile tool manufacture by providing a starting point for the first rip. In this way juveniles would learn to start tool manufacture at a suitable place (typically one third to midway along the leaf edge in adult tool manufacture) rather than at the thin and damaged leaf tips. Leaves of Pandanus sp. trees were often broken, or shortened by us if they impaired vision on the table. The high proportion of early juvenile tool manufacture starting at these damaged leaf edges (Figure 8) indicates that making the first cut into an intact leaf edge might be an important step in the development of tool manufacture. Counterparts could also guide juveniles about the ideal depth of a first cut into the pandanus leaf.

NC crow parents may also influence the emergence of juveniles' tool preferences via stimulus enhancement. Most adult crows have a strong, if not exclusive preference to manufacture and use either stick or pandanus tools (Hunt \& Gray, 2007). In the current study, all juveniles that developed a preference for pandanus tools had at least one parent with the same preference. We have also observed that two juveniles of a pair in which both partners preferred to use stick tools also developed a preference for stick tools (Holzhaider et al., unpublished data). While basic stick tool use can develop without social learning and may be the 'default' tool use for NC crows (Kenward et al., 2005, 2006; Hunt et al., 2007), our results are consistent with the idea that social input is necessary for the development of pandanus tool skills in the wild (Hunt \& Gray, 2003; Hunt et al., 2007). Only one crow in this study, Abel, failed to develop a preference for pandanus tools, in spite of both his father and sibling preferring to make and use wide pandanus tools (Table 3). While Abel had shown signs of turning into a pandanus tool user up to 6 months of age, he then switched to predominantly using stick tools. Surprisingly, however, he manufactured two adult-like pandanus tools at around 12 months. The most likely explanation for this abrupt emergence of adult-like wide tool manufacture is that most of Abel's learning took place away from the tables. From our earliest observations Abel's sibling Cain was clearly the dominant bird, frequently attacking and chasing 
Abel off, and even taking tools from his bill and then discarding them. Abel may have avoided competition from Cain for access to pandanus tools at the feeding tables and relied on the more easily accessible stick tools away from the table.

Discarded tools and other artefactual material such as counterparts might not only enable juveniles to obtain early experience of tool use and manufacture, and influence their emerging preference of tool type, but also provide a way of transferring cultural information about specific tool designs. Juvenile crows might use tools and counterparts on pandanus leaves as templates for their own tool manufacture. In this way the wide, narrow and stepped tool designs could be faithfully transmitted between generations even in the absence of imitation. Template matching is a well-described process in songbirds. During a sensitive period young birds hear and memorize a tutor song. By practicing themselves they then gradually match their own song to the memorized template (Nottebohm, 1984; Konishi, 1985; Doupe \& Konishi, 1991). By this mechanism songbirds faithfully transmit local song dialect traditions (Mundinger, 1980). A similar process of template matching might occur in the development of NC crow tool manufacture, with juveniles gradually adjusting the shape of their own tools to the designs manufactured by their parents.

A sensitive learning phase might also be involved in the acquisition of tool skills in NC crows. The fact that four out of five adult NC crows in the Nouméa zoo did not attempt to manufacture pandanus tools is consistent with this idea (Hunt et al., 2007). It seems likely that such a phase would occur within the first six months of a crow's life when it spends the majority of its time with its parents and has direct access to parental tools and counterparts. A sensitive period during this time might also serve to imprint juveniles with the tool designs manufactured by their parents and reduce the possibility of horizontal transmission. Similar age effects have been shown in the acquisition of tool use and special feeding techniques in other birds and mammals. For example, woodpecker finches have been suggested to have a sensitive learning phase during which they acquire tool skills (Tebbich et al., 2001). Similarly, wild chimpanzees who did not begin to crack nuts by the end of a sensitive phase between the ages of three and five years of age never learned that skill later in life (Matsuzawa, 1994; Biro et al., 2003).

The possibility of acquiring knowledge through niche construction and individual learning notwithstanding, many authors regard imitation as crucial 
for the faithful transmission of complex skills (Boyd \& Richerson, 1996; Tomasello, 1999). Studies on nonhuman primates emphasize the importance of direct observation in the acquisition of tool use. Chimpanzees are frequently reported to 'watch intently' when, for example, their mother is engaged in nut cracking (Inoue-Nakamura \& Matsuzawa, 1997; Biro et al., 2006). Moreover, both chimpanzees (Hirata \& Morimura, 2000; Biro et al., 2003) and capuchins (Ottoni et al., 2005) prefer to observe animals that are more skilled than themselves, enhancing both their scrounging payoffs and social learning opportunities. In humans, watching and 'paying attention' is clearly crucial in order to faithfully learn from a demonstrator. We found that juvenile crows have ample opportunity to observe their parents' tool skills. An important aspect in the master-apprenticeship theory of education (Matsuzawa et al., 2001) is the high tolerance shown to infants by their mothers and other members of the community. Similarly, crow parents are highly tolerant towards their own offspring, allowing them to observe their tool use close up and scrounge tools and even extracted meat. However, juveniles at feeding tables appeared to show limited interest in parental tool manufacture (Figure 2). The large peripheral field of vision of Corvus species makes it difficult to judge what they are actually observing (Emery \& Clayton, 2004). Nevertheless, young NC crows rarely paid obvious attention to their parents' pandanus tool manufacture or followed them into a tree where manufacture occurred. In contrast, they intently watched parents from close up when they were extracting meat from holes and, to a lesser extent, baited trees. However, our observations at feeding tables might have been biased because we separated the location of tool manufacture (the Pandanus sp. tree) from the location of food extraction (the baited $\log$ ). The juveniles usually just waited on the log where the food was for their parents to extract it. Moreover, feeding tables were probably highly salient as food sources so juveniles were more likely to stay on them even when food was also placed in the Pandanus sp. tree later in the study. Away from feeding tables juveniles might watch tool manufacture more frequently by following parents into Pandanus sp. trees where both tool manufacture and use occur. Despite the juveniles' apparent lack of attention, we found some evidence that they might have imitated certain parental motor patterns for tool manufacture. We identified three variants of wide pandanus tool manufacture used by adults (Video 3). While juveniles did not adopt their respective parents' preference, the distribution of the variants between the two families was very different (Figure 7). 
Variant A appeared to be the 'default' variant and was used by all crows to some degree. Variant B was strongly predominant in Pandora's family, while Variant $\mathrm{C}$ was almost exclusively used by Adam and his offspring Cain. It is, therefore, possible that the juveniles learned about these variants by watching their parents and developed a preference for one of the variants they observed frequently. However, because of the small sample size we cannot exclude the possibility that this correspondence is due to chance. Further field research is needed to establish whether specific tool manufacture variants are faithfully transmitted within families.

In summary, the ontogeny of wide pandanus tool manufacture and use is a lengthy process similar to that of tool use in primates. The first three to six months in a young crow's life appear to be the most crucial period for the acquisition of tool skills. Juveniles spend most of this time with their parents, during which they often watch them using and manufacturing tools, get fed at a high rate, and commonly use discarded tools. From seven months onwards most juveniles have acquired the technical ability to produce wide pandanus tools of an adult-like shape, even if they still lack the speed of adults to manufacture tools and extract meat. Individual trial and error learning appears to play a major role in the development of pandanus tool manufacture, but juveniles also have ample opportunity to socially learn from their parents in a variety of ways. Parents scaffold the juveniles' environment early in their tool development by leading them to Pandanus sp. trees where they provide discarded tools and counterparts. This 'epistemic niche construction' (Laland et al., 2000; Odling-Smee et al., 2003; Sterelny, 2006), might stimulate and facilitate early tool use and manufacture and enable the continuation of local design traditions. Future research should concentrate on experimental work to investigate the possibility of template matching and the existence of a sensitive learning phase in the crows' ontogeny.

\section{Acknowledgements}

We thank William Wadrobert for kindly allowing us to work on his family's land in Wabao District, Maré, and the Province des Iles Loyauté for permission to work on Maré. Mick Sibley prepared DVD versions of the footage. Noel Andrews, Lindsey Davidson, Roland Rehm, Mick Sibley and Alex Taylor assisted with data collection. We thank Roland Rehm for the production of Figure 4 and Katie Palmer for help with the coding of video footage. This research was funded by a grant from the New Zealand Marsden Fund (R.D.G. and G.R.H.). The research reported in this paper was approved by the University of Auckland Animal Ethics committee (approvals R231 and R375) and complies with the laws of New Caledonia. 


\section{References}

Altmann, J. (1974). Observational study of behaviour: sampling methods. - Behaviour 49: 227-267.

Beck, B. (1980). The use and manufacture of tools by animals. — Garland STPM Press, New York, NY.

Biro, D., Inoue-Nakamura, N., Tonooka, R., Yamakoshi, G., Sousa, C. \& Matsuzawa, T. (2003). Cultural innovation and transmission of tool use in wild chimpanzees: evidence from field experiments. - Anim. Cogn. 6: 213-223.

Biro, D., Sousa, C. \& Matsuzawa, T. (2006). Ontogeny and cultural propagation of tool use by wild chimpanzees at Bossou, Guinea: case studies in nut cracking and leaf folding. In: Cognitive development in chimpanzees (Matsuzawa, T., Tomonaga, M. \& Tanaka, M., eds). Springer, Tokyo, p. 476-508.

Boesch, C. \& Boesch, H. (1990). Tool use and making in wild chimpanzees. — Folia Primatol. 54: 86-99.

Boyd, R. \& Richerson, P.J. (1996). Why culture is common, but cultural evolution is rare. In: Evolution of social behaviour patterns in primates and man: a joint discussion meeting of the Royal Society and the British Academy. - Oxford University Press, Oxford.

Call, J., Carpenter, M. \& Tomasello, M. (2005). Copying results and copying actions in the process of social learning: chimpanzees (Pan troglodytes) and human children (Homo sapiens). - Anim. Cogn. 8: 151-163.

Call, J. \& Tomasello, M. (1994). The social learning of tool use by orangutans (Pongo pygmaeus). — Hum. Evol. 9: 297-313.

Connolly, K. \& Dalgleish, M. (1989). The emergence of a tool-using skill in infancy. — Dev. Psychol. 25: 894-912.

de Resende, B.D., Ottoni, E.B. \& Fragaszy, D.M. (2008). Ontogeny of manipulative behaviour and nut-cracking in young tufted capuchin monkeys (Cebus apella): a perceptionaction perspective. - Dev. Sci. 11: 828-840.

Doupe, A.J. \& Konishi, M. (1991). Song-selective auditory circuits in the vocal control system of the zebra finch. - Proc. Natl. Acad. Sci. USA 88: 11339-11343.

Emery, N.J. \& Clayton, N.S. (2004). Comparing avian and primate cognition. A case of divergent neurological evolution and convergent mental evolution? - In: Are primates special? (Rogers, L.J. \& Kaplan, G., eds). Kluwer, Dordrecht, p. 3-55.

Fragaszy, D.M. \& Adams-Curtis, L.E. (1997). Developmental changes in manipulation in Tufted Capuchins (Cebus apella) from birth through 2 years and their relation to foraging and weaning. - J. Comp. Psychol. 111: 201-211.

Fragaszy, D.M., Visalberghi, E. \& Fedigan, L. (2004). The complete capuchin. — Cambridge University Press, Cambridge.

Goodall, J. (1968). The behaviour of free-living chimpanzees in the Gombe Stream Reserve. — Anim. Behav. Monogr. 1: 163-211.

Gumert, M.D., Kluck, M. \& Malaivijitnond, S. (2009). The physical characteristics and usage patterns of stone axe and pounding hammers used by long-tailed macaques in the Andaman Sea region of Thailand. - Am. J. Primatol. 71: 594-608.

Hayashi, M., Mizuno, Y. \& Matsuzawa, T. (2005). How does stone-tool use emerge? Introduction of stones and nuts to naïve chimpanzees in captivity. - Primates 46: 91-102.

Heyes, C.M. (1993). Imitation, culture and cognition. — Anim. Behav. 46: 999-1010. 
Hirata, S. \& Celli, M. (2003). Role of mothers in the acquisition of tool-use behaviours by captive infant chimpanzees. - Anim. Cogn. 6: 235-244.

Hirata, S. \& Morimura, N. (2000). Naive chimpanzees' (Pan troglodytes) observation of experienced conspecifics in a tool-using task. — J. Comp. Psychol. 114: 291-296.

Holzhaider, J., Hunt, G., Campbell, V. \& Gray, R. (2008). Do wild New Caledonian crows (Corvus moneduloides) attend to the functional properties of their tools? - Anim. Cogn. 11: 243-254.

Horner, V. \& Whiten, A. (2005). Causal knowledge and imitation/emulation switching in chimpanzees (Pan troglodytes) and children (Homo sapiens). — Anim. Cogn. 8: 164181.

Humle, T. (2006). Ant dipping in chimpanzees: an example of how microecological variables, tool use, and culture reflect the cognitive abilities of chimpanzees. - In: Cognitive development in chimpanzees (Matsuzawa, T., Tomonaga, M. \& Tanaka, M., eds). Springer, Tokyo, p. 452-475.

Hunt, G.R. (1996). Manufacture and use of hook-tools by New Caledonian crows. - Nature 397: 249-251.

Hunt, G.R. (2000a). Human-like, population-level specialization in the manufacture of pandanus tools by New Caledonian crows Corvus moneduloides. — Proc. R. Soc. Lond. B: Biol. 267: 403-413.

Hunt, G.R. (2000b). Tool use by the New Caledonian crow Corvus moneduloides to obtain Cerambycidae from dead wood. - Emu 100: 109-114.

Hunt, G.R. \& Gray, R.D. (2002). Species-wide manufacture of stick-type tools by New Caledonian crows. - Emu 102: 349-353.

Hunt, G.R. \& Gray, R.D. (2003). Diversification and cumulative evolution in New Caledonian crow tool manufacture. — Proc. Roy. Soc. Lond. B: Biol. 270: 867-874.

Hunt, G.R. \& Gray, R.D. (2004a). The crafting of hook tools by wild New Caledonian crows. — Proc. R. Soc. Lond. B: Biol. 271: 88-90.

Hunt, G.R. \& Gray, R.D. (2004b). Direct observations of pandanus-tool manufacture and use by a New Caledonian crow (Corvus moneduloides). — Anim. Cogn. 7: 114-120.

Hunt, G.R. \& Gray, R.D. (2007). Parallel tool industries in New Caledonian crows. - Biol. Lett. 3: 173-175.

Hunt, G.R., Lambert, C. \& Gray, R.D. (2007). Cognitive requirements for tool use by New Caledonian crows - N.Z.J. Zool. 34: 1-7.

Inoue-Nakamura, N. \& Matsuzawa, T. (1997). Development of stone tool use by wild chimpanzees (Pan troglodytes). - J. Comp. Psychol. 111: 159-173.

Kenward, B., Rutz, C., Weir, A.A.S. \& Kacelnik, A. (2006). Development of tool use in New Caledonian crows: inherited action patterns and social influences. - Anim. Behav. 72: 1329-1343.

Kenward, B., Weir, A.A.S., Rutz, C. \& Kacelnik, A. (2005). Behavioural ecology: tool manufacture by naive juvenile crows. - Nature 433: 121-121.

Konishi, M. (1985). Birdsong: from Behaviour to Neuron. - Annu. Rev. Neurosci. 8: 125170.

Laland, K.N. \& Hoppitt, W. (2003). Do animals have culture? - Evol. Anthropol. Issues News Rev. 12: 150-159.

Laland, K.N., Odling-Smee, J. \& Feldman, M.W. (2000). Niche construction, biological evolution, and cultural change. - Behav. Brain Sci. 23: 131-146. 
Lockman, J.J. (2000). A perception-action perspective on tool use development. — Child Dev. 71: 137-144.

Matsuzawa, T. (1994). Field experiments on use of stone tool by chimpanzees in the wild. In: Chimpanzee cultures (Wrangham, R.W., McGrew, W.C., de Waal, F.B.M. \& Heltne, P., eds). Harvard University Press, Cambridge, MA, p. 351-370.

Matsuzawa, T., Biro, D., Humle, T., Inoue-Nakamura, N., Tonooka, R. \& Yamakoshi, G. (2001). Emergence of culture in wild chimpanzees: education by master-apprenticeship. - In: Primate origins of human cognition and behaviour (Matsuzawa, T., ed.). Springer, Tokyo, p. 557-574.

McGrew, W.C. (1974). Tool use by wild chimpanzees in feeding upon driver ants. - J. Hum. Evol. 3: 501-508.

McGrew, W.C., Tutin, C.E.G. \& Baldwin, P.J. (1979). Chimpanzees, tools, and termites: cross-cultural comparisons of Senegal, Tanzania, and Rio Muni. - Man 14: 185-214.

Mundinger, P.C. (1980). Animal cultures and a general theory of cultural evolution. - Ethol. Sociobiol. 1: 183-223.

Nagell, K., Olguin, R. \& Tomasello, M. (1993). Processes of social learning in the tool use of chimpanzees (Pan troglodytes) and human children (Homo sapiens). - J. Comp. Psychol. 107: 174-186.

Nishida, T. \& Hiraiwa, M. (1982). Natural history of a tool-using behaviour by wild chimpanzees in feeding upon wood-boring ants. - J. Hum. Evol. 11: 73-99.

Nottebohm, F. (1984). Birdsong as a model in which to study brain processes related to learning. - Condor 86: 227-236.

Odling-Smee, J., Laland, K.N. \& Feldman, M.W. (2003). Niche construction: the neglected process in evolution. - Princeton University Press, Princeton, NJ.

Ottoni, E. \& Izar, P. (2008). Capuchin monkey tool use: overview and implications. - Evol. Anthropol. Issues News Rev. 17: 171-178.

Ottoni, E., Resende, B. \& Izar, P. (2005). Watching the best nutcrackers: what capuchin monkeys (Cebus apella) know about others' tool-using skills. - Anim. Cogn. 8: 215219.

Reisman, K. (2007). Is culture inherited through social learning? - Biol. Theor. 2: 300-306.

Rochat, P. (2001). The infants' world. — Harvard University Press, Cambridge, MA.

Sterelny, K. (2006). The evolution and evolvability of culture. - Mind Language 21: 137165.

Takeshita, H., Fragaszy, D., Mizuno, Y., Matsuzawa, T., Tomonaga, M. \& Tanaka, M. (2005). Exploring by doing: how young chimpanzees discover surfaces through actions with objects. - Infant Behav. Dev. 28: 316-328.

Tebbich, S., Taborsky, M., Fessl, B. \& Blomqvist, D. (2001). Do woodpecker finches acquire tool-use by social learning? — Proc. Roy. Soc. Lond. B: Biol. 268: 2189-2193.

Thelen, E. \& Smith, L.B. (1994). A dynamic systems approach to the development of cognition and action. - MIT Press, Cambridge, MA.

Thouless, C.R., Fanshawe, J.H. \& Bertram, B.C.R. (1989). Egyptian vultures Neophron percnopterus and ostrich Struthio camelus eggs: the origins of stone throwing behaviour. - Ibis 131: 9-15.

Tomasello, M. (1999). The human adaptation for culture. — Annu. Rev. Anthropol. 28: 509529.

Tomasello, M., Davis-Dasilva, M., Camak, L. \& Bard, K. (1987). Observational learning of tool-use by young chimpanzees. - Hum. Evol. 2: 175-183. 
van Schaik, C.P., Ancrenaz, M., Borgen, G. \& Galdikas, B. (2003). Orangutan cultures and the evolution of material culture. - Science 299: 102-105.

Visalberghi, E. (1987). Acquisition of nut-cracking behaviour by two capuchin monkeys (Cebus apella). - Folia Primatol. 49: 168-181.

Want, S.C. \& Harris, P.L. (2001). Learning from other people's mistakes: causal understanding in learning to use a tool. — Child Dev. 72: 431-443.

Want, S.C. \& Harris, P.L. (2002). How do children ape? Applying concepts from the study of non-human primates to the developmental study of 'imitation' in children. - Dev. Sci. 5: $1-14$.

Whiten, A., Goodall, J., McGrew, W.C., Nishida, T., Reynolds, V., Sugiyama, Y., Tutin, C.E.G., Wrangham, R.W. \& Boesch, C. (1999). Cultures in chimpanzees. — Nature 399: 682-685.

Whiten, A., Goodall, J., McGrew, W.C., Nishida, T., Reynolds, V., Sugiyama, Y., Tutin, C.E.G., Wrangham, R.W. \& Boesch, C. (2001). Charting cultural variation in chimpanzees. - Behaviour 138: 1481-1516.

Whiten, A., McGuigan, N., Marshall-Pescini, S. \& Hopper, L.M. (2009). Emulation, imitation, over-imitation and the scope of culture for child and chimpanzee. - Phil. Trans. Roy. Soc. B 364: 2417-2428.

\section{Appendix}

The videos referred to in this Appendix can be seen at http://media.brill.nl/behaviour/147/5-6/

Video 1. Development of proficient pandanus tool use in baited logs.

Clip 1: Adam extracts a piece of meat from a baited log at a feeding table. He then feeds the meat to Cain (then about 5 months of age), who has been watching the extraction.

Clip 2: Wrong angle probing: Primo (ca. 2 months old). He tries to insert a tool into a hole but fails because he holds the tool at a wrong angle (note that Primo is using a tool that has been just dropped by his mother Pandora).

Clip 3: Folding: Primo (ca. 4 months old) takes a tool that is already in a hole and twists and bends it without actually probing.

Clip 4: Defective probing: Abel (ca. 4 months old) inserts a tool with both ends pointing upwards out of the hole, and then probes.

Video 2. Juveniles' pandanus tool manufacture techniques. This video shows the five different techniques of tool manufacture used by juveniles during their ontogeny to proficient pandanus tool manufacturers.

Clip 1: Technique 0: Abel (ca. 4 months old) rips in a non coordinated fashion at a pandanus leaf hanging onto the feeding table, but does not manage to remove a tool.

Clip 2: Technique 1: Primo (ca. 5 months old) removes a piece of leaf by ripping uncoordinatedly at a pandanus leaf. Note that both in Techniques 1 and 2 the birds start their ripping at a damaged leaf edge. 
Clip 2: Technique 2: Bo (ca. 8 months old) attempts to manufacture a tool with a coordinated cut-rip/cut-rip action. Because the second action cuts into the leaf more deeply than the first one, the rips do not align and the tool cannot be removed from the leaf.

Clip 3: Technique 3: Primo (ca. 8 months old) manufactures a tool using a coordinated cut-rip/cut-rip action. However, because the second cut-rip starts closer to the leaf base than the first one, Primo removes the tool holding it with the barbs pointing downwards.

Clip 4: Technique 4: Primo (ca. 18 months old) proficiently manufactures a pandanus tool, and uses it to extract meat from a baited Pandanus sp. tree.

Video 3. The three variants of adult-like pandanus tool manufacture. This video shows the three different variants of pandanus tool manufacture which may occur whenever a crow manufactures a tool with coordinated cut/rip actions. For better visibility, all tool manufactures are shown at $1 / 3$ of the original speed.

Clip 1: Variant A is used by all birds to a certain extent. Adam manufactures a pandanus tool with two cut-rip actions that join about half way along the tool.

Clip 2: Variant B was predominantly used by families 1 and 2 (Pandora, Abraxas, Primo and Yor). Abraxas makes a cut that is followed by a rather long rip. The second cut removes the tool from the leaf, without ripping towards the first cut-rip.

Clip 3: Variant $\mathrm{C}$ was mainly used by family 3 (Cain and Abel). Cain (ca. 9 months old) makes an initial cut, then a long cut-rip that joins the first cut. 